\title{
Energy harvesting textiles for a rainy day: woven piezoelectrics based on melt-spun PVDF microfibres with a conducting core
}

\author{
Anja Lund ${ }^{1,2}$, Karin Rundqvist ${ }^{2}$, Erik Nilsson ${ }^{3}$, Liyang $\mathrm{Yu}^{1}$, Bengt Hagström ${ }^{3,4}$ and Christian Müller (10
}

Recent advances in ubiquitous low-power electronics call for the development of light-weight and flexible energy sources. The textile format is highly attractive for unobtrusive harvesting of energy from e.g., biomechanical movements. Here, we report the manufacture and characterisation of fully textile piezoelectric generators that can operate under wet conditions. We use a weaving loom to realise textile bands with yarns of melt-spun piezoelectric microfibres, that consist of a conducting core surrounded by $\beta$ phase poly(vinylidene fluoride) (PVDF), in the warp direction. The core-sheath constitution of the piezoelectric microfibres results in a-for electronic textiles-unique architecture. The inner electrode is fully shielded from the outer electrode (made up of conducting yarns that are integrated in the weft direction) which prevents shorting under wet conditions. As a result, and in contrast to other energy harvesting textiles, we are able to demonstrate piezoelectric fabrics that do not only continue to function when in contact with water, but show enhanced performance. The piezoelectric bands generate an output of several volts at strains below one percent. We show that integration into the shoulder strap of a laptop case permits the continuous generation of four microwatts of power during a brisk walk. This promising performance, combined with the fact that our solution uses scalable materials and well-established industrial manufacturing methods, opens up the possibility to develop wearable electronics that are powered by piezoelectric textiles.

npj Flexible Electronics (2018)2:9; doi:10.1038/s41528-018-0022-4

\section{INTRODUCTION}

Textiles are an ideal format for low-power electronics that promise to enrich every aspect of life from fashion, functional clothing and medical care to interior design and technical textiles. Electronic textiles (e-textiles) are poised to play a key role in the development of the "Internet of Things", connecting an ever larger number of objects to each other and to the cloud. ${ }^{1,2} \mathrm{~A}$ wide range of e-textile functionalities have been demonstrated, ranging from input devices such as keyboards ${ }^{3-5}$ and sensors, ${ }^{6-9}$ to electronic circuitry ${ }^{10,11}$ that can process and store data. Moreover, textiles can interact with the user as displays based on electrochromic or electroluminescent fibres, ${ }^{12,13}$ or even via movement as artificial muscles. ${ }^{14,15}$ For e-textiles to function as comfortable and maintenance-free wearables, batteries should be replaced with energy harvesting devices. These can draw from external energy sources, such as the sun, e.g., through fibreshaped solar cells. ${ }^{16,17}$ Alternatively, excess energy from the immediate environment can be utilised, e.g., in the form of body heat and motion by exploiting the thermoelectric, ${ }^{18-21}$ triboelectric $^{22-24}$ or piezoelectric effect. ${ }^{25-27}$ For widespread use, etextile generators should be fabricated with processing methods that are familiar to the textile industry. Coupled with recent developments in energy storage in the form of knitted and woven supercapacitors, ${ }^{28-31}$ textiles can then function as an autonomous power source.

A particularly promising concept is the use of piezoelectric materials, which are able to transform minimal movement into a large electrical potential. For textile applications, piezoelectric polymers are the most suitable choice due to their low weight in combination with outstanding flexibility and tensile strength. Poly (vinylidene fluoride) (PVDF) offers the highest piezoelectric coefficient, provided that one of the polar crystal phases can be accessed. ${ }^{32}$ The polymorphic nature of PVDF allows the polymer to crystallise in at least four different phases, known as the $a, \beta, \gamma$ and $\delta$ phase. ${ }^{33}$ Out of these, the $\beta$ phase shows the highest polarisation ${ }^{34}$ resulting in an electromechanical coupling coeffiecient of at least $11 \% .{ }^{33}$ The $\beta$ phase can be induced by a variety of means including - for fibre and textile production relevant-colddrawing. The ability of PVDF to convert biomechanical energy to electrical power has been explored in a variety of architectures, ranging from blocks in shoe soles ${ }^{35}$ and tapes ${ }^{36}$ to electrospun fibres. $^{37-40}$ Moreover, melt-spun PVDF fibres have been considered for energy harvesting. For instance, Soin et al. reported a 3Dspacer textile, which was able to produce a peak power output exceeding $1 \mu \mathrm{W} / \mathrm{cm}^{2}$ at an impact pressure of $20 \mathrm{kPa}^{41}$

A prevailing question in discussions related to wearable electronics and all types of e-textiles is "But can you wash them?" Although it would probably be acceptable to label a high-end functional textile as "dry clean only", ${ }^{30}$ it also seems fair to expect that a textile should retain its main functionality even after being subjected to sweat, ambient humidity or rain. A major disadvantage of many previously described textile generators-piezoelectric as well as tribo- and thermoelectric-is that both electrodes are exposed to the surroundings. Consequently, they are susceptible to short-circuiting caused by high humidity ${ }^{22}$ and

\footnotetext{
${ }^{1}$ Department of Chemistry and Chemical Engineering, Chalmers University of Technology, Göteborg 412 96, Sweden; ${ }^{2}$ The Swedish School of Textiles, University of Borås, Borås

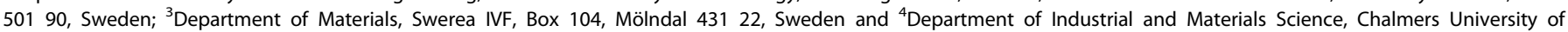
Technology, Göteborg 412 96, Sweden

Correspondence: Anja Lund (anja.lund@chalmers.se) or Christian Müller (christian.muller@chalmers.se)
}

Received: 24 September 2017 Revised: 28 December 2017 Accepted: 11 January 2018

Published online: 22 March 2018 
will cease to function under practical conditions. Furthermore, many previously presented e-textile generators were largely handcrafted and would prove difficult to realise on a large scale and to implement in everyday life.

Here, we present an energy generating piezoelectric textile where the power output is enhanced by water. Our design is based on melt-spun continuous microfibres with a core-sheath structure, where one electrode is hidden within the core of the fibre. Gratifyingly, the robust and wear-resistant nature of these fibres allows us to exploit traditional manufacturing routes. To complete the device, the second electrode in the form of a conducting yarn is wrapped around yarns of bicomponent microfibres by weaving. We demonstrate machine woven textile bands with a promising piezoelectric power-harvesting functionality, generating an output voltage of $3.5 \mathrm{~V}$ when experiencing a sinusoidal axial strain of $0.25 \%$ at a low frequency of $4 \mathrm{~Hz}$. To demonstrate a piezoelectric textile for practical every-day energy harvesting, a $2.5 \mathrm{~cm} \times 20 \mathrm{~cm}$ piece of this textile was integrated (sewn) into the shoulder strap of a laptop case. The piezoelectric textile thus constitutes a load-bearing component that also provides an output voltage of up to $8 \mathrm{~V}$ per step under wet conditions, as water enhances the electrical contact surface area between fibres. A brisk walk with the case produced a continuous output of $4 \mu \mathrm{W}$. The influence of materials selection and processing conditions on the resulting e-textile properties is thoroughly discussed, and routes are outlined to further improve the performance of our piezoelectric textile generators.

\section{RESULTS}

Melt spun bicomponent microfibres with a conducting core For bicomponent fibre spinning, the constituent materials are melted in two separate extruders, and fed in parallel through a multichannel spinneret with 24 holes, from which the two materials are coextruded. This produces 24 filaments, i.e., virtually endless fibres, each with a coaxial core/sheath configuration (Fig. 1a). Melt spinning was carried out in a pilot scale spinning equipment, with an extrusion rate $v_{\mathrm{e}}=5.3 \mathrm{~m} / \mathrm{min}$ and take up rate $v_{\mathrm{t}}=116 \mathrm{~m} / \mathrm{min}$, resulting in a substantial degree of stretch alignment in the molten state. The final yarn winding speed of $v_{\mathrm{w}}=500 \mathrm{~m} / \mathrm{min}$ provides an inline cold drawing ratio $\lambda=v_{\mathrm{w}} / v_{\mathrm{t}}=$ 4.3. Cold drawing is a critical part of any melt spinning process, as it is to a large extent responsible for the tenacity and elasticity of the final fibres. ${ }^{42}$ In the case of PVDF, cold drawing additionally converts the non-polar a-crystals to the polar $\beta$ phase. ${ }^{43,44}$ Figure $1 \mathrm{~b}$ displays a comparison of transmission wide-angle $\mathrm{X}$-ray scattering (WAXS) patterns (Fig. 1b left) of the PVDF pellets-the raw material-and fibres. A number of concentric diffraction rings can be observed in the WAXS patterns of the pellets, indicating an isotropic distribution of crystallites. After spinning, the PVDF develops a preferred orientation resulting in a clear diffraction perpendicular to the fibre axis. The anisotropy of the PVDF sheath is also supported by birefringence that is observed when placing the fibres between crossed polarisers (Fig. 1c). The integrated cake slices (Fig. 1b right) along the horizon of the two patterns provide

a

b
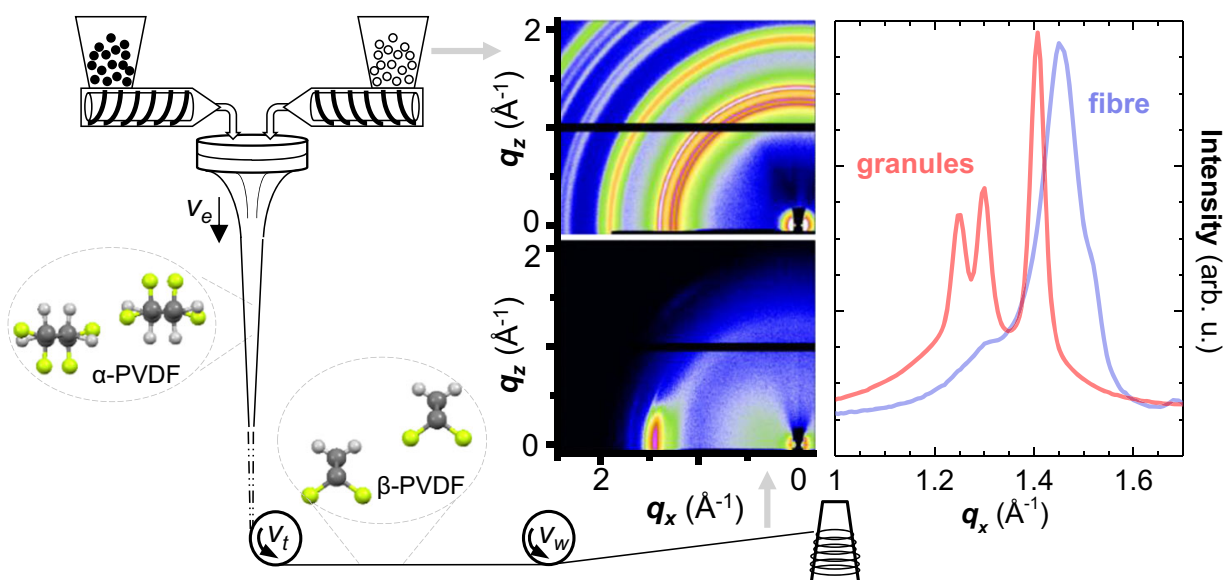

C

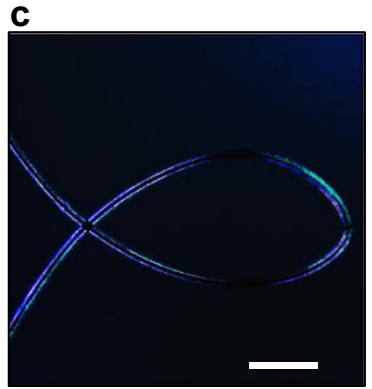

d

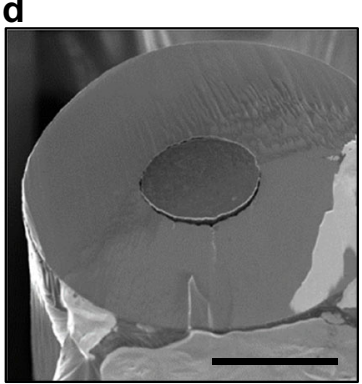

e

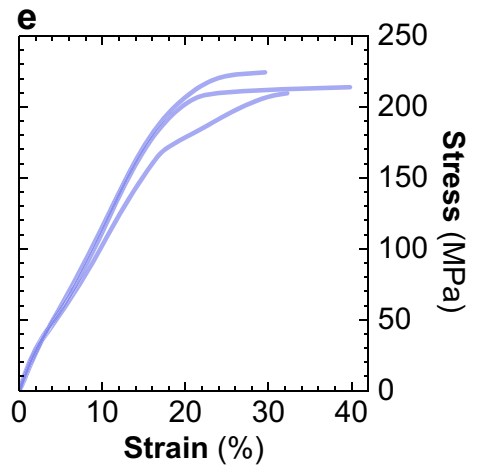

Fig. 1 Melt spinning and structural characterisation of bicomponent filaments. a Schematic of bicomponent fibre spinning: the base materials for the core (carbon black/polyethylene) and sheath (PVDF) are fed from two separate extruders through the spinneret, producing a yarn of 24 bicomponent filaments $\left(v_{\mathrm{e}}=5.3 \mathrm{~m} / \mathrm{min} ; v_{\mathrm{t}}=116 \mathrm{~m} / \mathrm{min}\right.$; melt draw ratio $\left.v_{t} / v_{\mathrm{e}}=22\right)$. The second cold-drawing stage $\left(v_{\mathrm{w}}=500 \mathrm{~m} / \mathrm{min}\right.$; cold draw ratio $v_{\mathrm{w}} / v_{\mathrm{t}}=4.3$ ) converts the PVDF from $\alpha$ to $\beta$ phase. b Transmission wide-angle X-ray scattering (WAXS) patterns (left) and diffractograms (right) of PVDF granules (top pattern and red line) and the final bicomponent filaments (bottom pattern and blue line); c crosspolarised optical micrograph of a single filament, scale bar is $200 \mu \mathrm{m}$. d Scanning electron microscopy (SEM) image of the cross-section of a single filament, scale bar is $20 \mu \mathrm{m}$. e Stress-strain behaviour of three samples of bicomponent yarns during tensile drawing 
additional information on the crystalline order. In case of the granules three peaks can be distinguished that correspond to the (100), (020) and (110) diffraction of a-crystalline PVDF. In clear contrast, the diffraction pattern of the fibre consists of a main peak attributed to both the (200) and (110) diffraction of $\beta$-crystalline PVDF. ${ }^{45,46}$ This peak features two shoulders, one at a lower $q_{\mathrm{x}}$ value that suggests some residual a-crystalline PVDF, and one at a higher $q_{\mathrm{x}}$ value that is due to the polyethylene core (see further Fig. S1, Suppl. Information).

The conducting core of the bicomponent fibres consists of 10 wt $\%$ carbon black in a polyethylene matrix. Already at a few percent loading of carbon black, rheological percolation occurs causing a notable increase in the shear viscosity of the carbon black/polyethylene compound. ${ }^{47}$ At higher loadings the compound tends to develop a pronounced elastic behaviour in the melt, which complicates fibre spinning as it causes spin-line instability. ${ }^{48}$ The spinnability can be enhanced by using coextrusion. In our system the outer PVDF layer bears the major share of the spin-line force while maintaining a coaxial configuration. The cross-section of the bicomponent fibres displays a well-defined circular core in close contact with the sheath (Fig. 1d). This configuration is promoted by the differences in volumetric contraction, i.e., the decrease in specific volume, upon cooling from the extrusion temperature of $240{ }^{\circ} \mathrm{C}$ to room temperature. Typically, the contraction in PVDF and polyethylene are in the same range (18.9 and $18.8 \%$, respectively $\left.{ }^{49}\right)$ and in our material the addition of $10 \mathrm{wt} \%$ carbon black to the polyethylene will further reduce the volume contraction of the core compound, favouring a close contact between the sheath and core in the final fibre. To counter the negative effect of fibre drawing on the percolation, and hence on the electrical conductivity of the inner carbon black/polyethylene core, we annealed the bicomponent fibres in-line during fibre spinning, at $120^{\circ} \mathrm{C}$ (i.e., at the melting temperature $T_{\mathrm{m}}$ of polyethylene but well below the $T_{\mathrm{m}}$ of PVDF). Our final multifilament yarn is constituted of the 24 filaments. The single filaments have an average diameter of $60 \mu \mathrm{m}$, a core diameter of $24 \mu \mathrm{m}$ and a linear density of $40 \mathrm{dtex}$ (dtex = weight in grams per $10,000 \mathrm{~m}$ ). The core conductivity is $0.2 \mathrm{~S} / \mathrm{cm}$. We deduce a tenacity of about $225 \mathrm{MPa}$ and a strain to break $~ 30 \%$ with a yield point around $220 \mathrm{MPa}$ and $20 \%$ strain (Fig. 1e).

\section{Piezoelectric characteristics of single yarns}

For initial piezoelectric characterisation, silver paste was applied to the outside of the yarns along lengths $L=10$ and $20 \mathrm{~mm}$ of the yarns, to constitute the outer electrode (Fig. 2a). The yarns were contact poled by applying an electric field over its two electrodes via crocodile clips, resulting in a piezoelectric yarn-a piezoyarn (Fig. S2, Suppl. Information). We have previously calculated the piezoelectric constant $g_{31}$ of the piezoyarns to be about $0.3 \mathrm{~V} / \mathrm{m} /$ $\mathrm{Nm}^{2},{ }^{50}$ where the electrical axis (3) is a polar axis taken as parallel to the radius of a single fibre, and the mechanical axis (1) is taken as parallel to the fibre axis (see further Fig. S2, Suppl. Information). We demonstrated that the yarns can function as sensors for heartbeat and respiration ${ }^{50}$ and for motions in e.g., finger joints. ${ }^{51}$ Here, to elucidate the relationship between the applied deformation and generated voltage, the yarn was placed in a dynamic mechanical analyser set to apply a dynamic (sinusoidal) tensile strain with the strain amplitude ramped from 0.01 to $0.45 \%$, under a pre-load of $0.5 \mathrm{~N}$ and at a frequency $f=10 \mathrm{~Hz}$. This range of deformation is within the elastic region of the yarns (Fig. 1e). The generated piezoelectric voltage increased linearly with the applied axial strain (Fig. 2b), and provided a voltage amplitude of $1 \mathrm{~V}$ at less than $0.5 \%$ strain. Next, we carried out a frequency sweep at a dynamic strain of amplitude $\varepsilon=0.1 \%$. Just like the applied mechanical strain, the generated voltage signal is sinusoidal (Fig. 2c). The voltage amplitude displays a frequency dependence and a
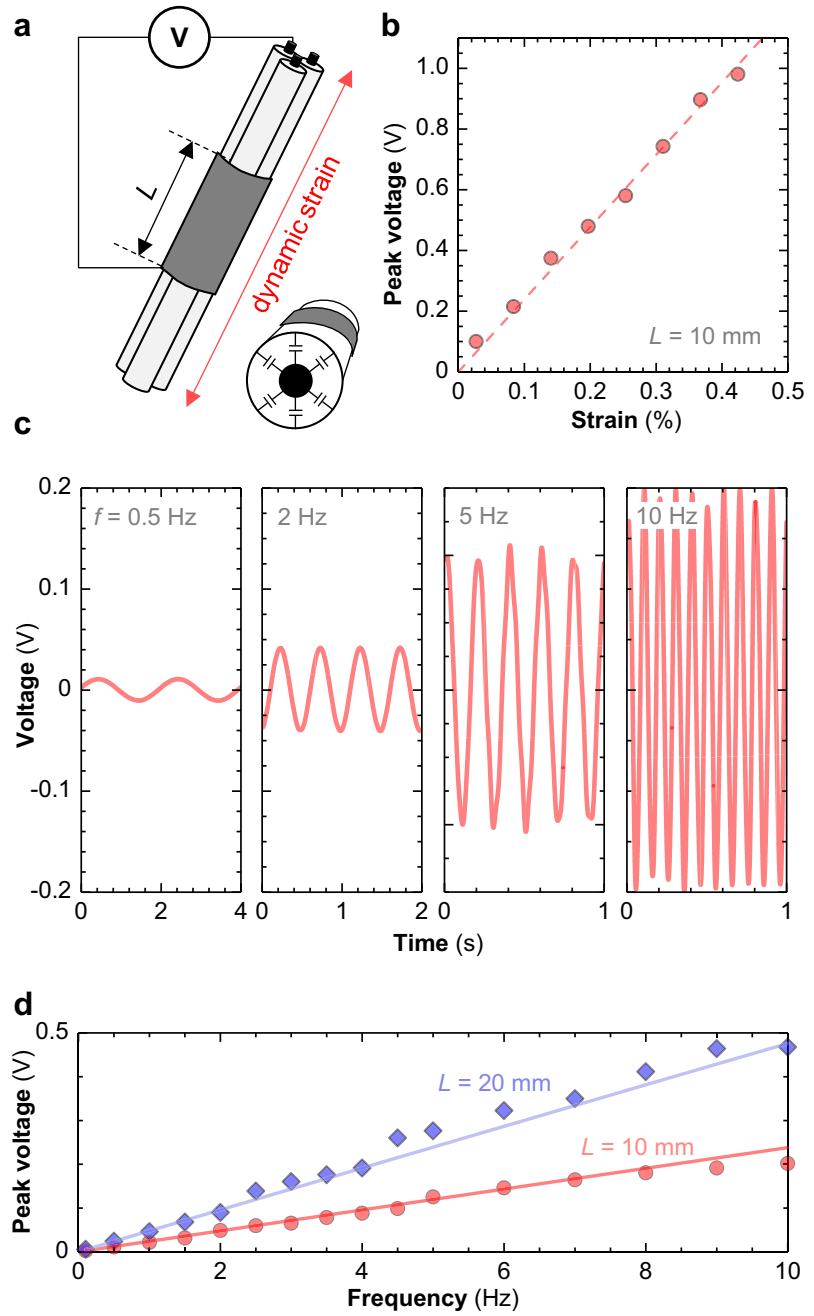

e
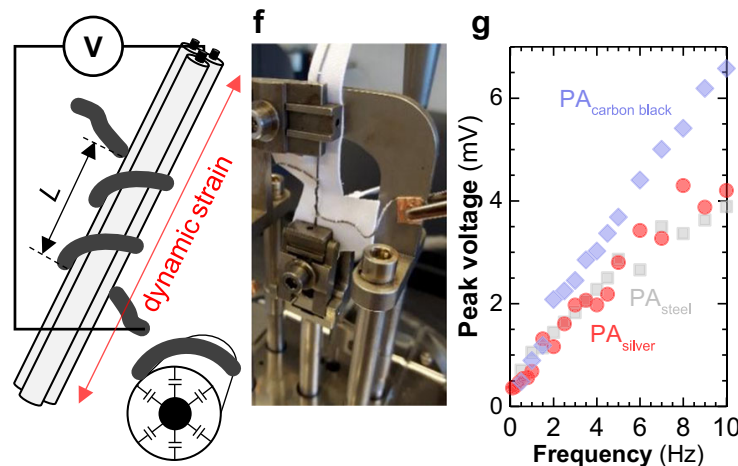

Fig. 2 Piezoelectric characterisation of single piezoyarns. a Schematic of a piezoyarn with silver paste applied over a length $L$. For clarity, only 3 filaments are depicted instead of all 24. b Piezoelectric output at a dynamic strain frequency of $f=10 \mathrm{~Hz}$ as a function of peak dynamic strain $\varepsilon(L=10 \mathrm{~mm})$. c Sinusoidal piezoelectric voltage for $f=0.5,2,5$ and $10 \mathrm{~Hz}(\varepsilon=0.1 \%)$. d Measured (symbols) and calculated (solid lines) piezoelectric peak voltage as a function of frequency for $L=10 \mathrm{~mm}$ (red circles) and $20 \mathrm{~mm}$ (blue diamonds). e Schematic illustration of a piezoyarn with a conducting yarn as the outer electrode. For clarity, only 3 bicomponent filaments are depicted instead of all 24. $f$ A piezoyarn with a $\mathrm{PA}_{\text {silver }}$ conducting yarn as the outer electrode mounted in a dynamic mechanical analysis (DMA) instrument. g Generated peak voltage as a function of frequency for the piezoyarn with a conducting yarn as the outer electrode: $\mathrm{PA}_{\text {carbon black }}$ (blue diamonds), $\mathrm{PA}_{\text {steel }}$ (grey squares), and $\mathrm{PA}_{\text {silver }}$ (red circles) 
increases linearly with frequency in the range of $f=1-10 \mathrm{~Hz}$ (Fig. $2 d$ ); a range relevant for human motion. ${ }^{52}$

The frequency dependence can be ascribed to the typical high pass filter characteristics of a piezoelectric circuit, where the generated voltage increases with increasing frequency up to the cut-off frequency $f_{c}$, and levels out at $f>f_{c}$. As a polarised PVDFlayer is compressed and released its charge density varies, resulting in an alternating voltage potential over its electrodes. Closing the circuit allows a current flow to neutralise the potential fluctuations. The current flow rate is set by the source potential, the inherent capacitance and the external circuitry. Thus, a piezoelectric element of PVDF sandwiched between two electrodes can be described as an AC voltage supply providing a voltage amplitude $V_{\mathrm{i}}$ in series with an inherent capacitance $C_{i}$ (cf. Figure 4a). For the piezoyarn a resistance $R_{\mathrm{i}}$ of substantial value, corresponding to the non-metal electrodes, also adds to the circuit. Note that the inherent AC-voltage of the piezoelectric element is not measurable in reality, as the voltage source is physically inseparable from its inherent capacitance. We measured the values of $R_{\mathrm{i}}$ and $C_{\mathrm{i}}$ using a standard LCR-instrument. The internal impedance $Z_{\mathrm{i}}$ is given by:

$$
\left|Z_{i}\right|=\sqrt{R_{i}^{2}+\left(2 \pi f C_{i}\right)^{-2}}
$$

A correct model of the piezoelectric performance needs to also take the measurement device (here an oscilloscope) into account, as it in this case constitutes a non-negligible load. The oscilloscope impedance is derived from its $R_{\text {input }}=10 \mathrm{M} \Omega$ and $C_{\text {input }}=13 \mathrm{pF}$, according to Equation 1. Combining this with the measured values for the piezoyarn (Table S1, Suppl. Information), the frequency behaviour of the piezoyarns was modelled using an open-source SPICE simulation software (see Methods section for details). The software-modelled behaviour, plotted as solid lines in Fig. 2d, accurately describes the experimental data. The length of the outer silver paste electrode determines the active piezoelectric area and, correspondingly, the supplied voltage amplitude $V_{i}$ of the model linearly scales with the covered length $L$ (Fig. $2 \mathrm{~d}$ ). The predicted $f_{c}$, defined as the frequency where $V=V_{\max } / \sqrt{2}$, is above $100 \mathrm{~Hz}$ for a single piezoyarn $(L=10 \mathrm{~mm})$ for this particular system, i.e., with the oscilloscope as load (Fig. S3a, b, Suppl. Information). For a $10 \mathrm{~mm}$ long single yarn we predict that impedance matching where $R_{\text {load }}=\left|Z_{\mathrm{i}}\right|$ gives rise to an output power of $23 \mathrm{nW} / \mathrm{cm}$ or a specific power of $23 \mu \mathrm{W} / \mathrm{g}$ (the piezoyarn weighs approximately $1 \mathrm{mg}$ per cm length) at $10 \mathrm{~Hz}$ (Fig. S3c, Suppl. Information).

In a further set of experiments we explored the use of alternative outer electrodes that are more applicable to textiles and their manufacture. We replaced silver paste, which is prone to brittle failure, with conducting yarns (Fig. 2e). For this system, we evaluated three types of commercial conducting yarns: a polyamide yarn coated with carbon black-particles $\left(\mathrm{PA}_{\text {carbon black }}\right)$, a polyamide yarn commingled with $20 \%$ thin stainless steel staple fibres $\left(\mathrm{PA}_{\text {steel }}\right)$, and a silver plated polyamide yarn $\left(\mathrm{PA}_{\text {silver }}\right)$. The yarns were wrapped around the piezoyarn (two turns per $10 \mathrm{~mm}$ length). Dynamic electromechanical analysis indicates that the output voltage increases with frequency, reaching peak values at $f$ $=10 \mathrm{~Hz}$ of $7 \mathrm{mV}\left(\mathrm{PA}_{\text {carbon black }}\right), 4.25 \mathrm{mV}\left(\mathrm{PA}_{\text {silver }}\right)$ and $3.9 \mathrm{mV}$ $\left(\mathrm{PA}_{\text {steel }}\right)$ (Fig. 2f, g). Clearly, the conducting yarns are functioning as outer electrodes. As expected the lower interfacial area between the piezoyarn and outer electrode, compared to the silver paint electrode, is reflected in the measurable voltage output. Increasing the number of turns per $10 \mathrm{~mm}$ would increase the covering area of the electrode, and consequently increase the measurable voltage output. A more efficient way to combine the yarns is to make a textile from them. All three yarns were further evaluated for weaving of piezoelectric textiles.

\section{Fabrication of piezoelectric textiles}

To produce a robust textile for energy harvesting we used an industrial-type band weaving machine (Fig. 3a). A total of 60 piezoyarns in parallel were inserted as the warp. The conducting yarns were inserted as the weft, thus constituting a flexible outer electrode. The woven bands were poled using a corona method (Fig. S4 and Table S2, Suppl. Information), which is convenient for large scale fabrication. ${ }^{53}$ The bands show a pronounced piezoelectric effect when stretched (Fig. 3b and Fig. S5a, Suppl. Information). Moreover, the piezoelectric behaviour persists when the whole strap is submerged in tap water (Fig. 3c). Note that we held the textile with bare hands. Since we only touch the outer electrode, no short-circuiting occurs and the functionality of the piezoelectric textile is not compromised.

We anticipate that the weave construction will influence both the mechanical properties and the coverage i.e., the relative area of the fabric that is covered by one set of the yarns (warp or weft). These properties in turn are likely to affect the piezoelectric behaviour. Three different weave architectures were evaluated: plain weave (Fig. 3d), twill (Fig. 3e) and weft rib (Fig. S5b, Table S3, Suppl. Information). A plain weave fabric is mechanically stable, as this weave construction maximises the number of intersections between the warp and weft yarns. It also results in a high degree of crimp, or waviness, in the warp yarns (Fig. 3d right). The twill construction includes flotations (i.e., unbound stretches of yarn) that provide a lower degree of crimp in the warp yarn (Fig. 3e right). The front surface of the twill fabric is dominated by the warp yarns and the back surface is dominated by the weft (Fig. S5c, Suppl. Information). The piezoelectric effect in woven bands was characterised by dynamically stretching them to a strain amplitude $\varepsilon=0.25 \%$ at $f=4 \mathrm{~Hz}$. The pre-load was $30 \mathrm{~N}$, which is sufficient to remove most of the crimp (Fig. S6, Suppl. Information). The generated output voltage follows the sinusoidal deformation mode. As in the single yarns, the piezoelectric voltage is generated over a polar axis between the fibre core and the fibre perimeter. The measurable voltage can be directly related to the presence of an overlapping outer electrode. Consequently, we observe that both the type of weft yarn and the weave construction influence the output voltage. The weft rib construction gave rise to a low and erratic signal (Fig. S7, Suppl. Information). We rationalise this behaviour with the high cover factor of $50 \%$, which may in fact have a detrimental effect on the poling, as the outer electrode in the weft rib can act as an electromagnetic shield reducing the effect from corona treatment. The twill construction resulted in the highest generated voltage, independent of the choice of conducting yarn for the outer electrode (Fig. 3f, g and Fig. S8, Suppl. Information). The bands with $\mathrm{PA}_{\text {steel }}$-weft and $\mathrm{PA}_{\text {silver }}$-weft yarns gave rise to the highest output voltage in both plain weave and twill.

We chose to characterise the twill-textiles in more detail. Notably, the frequency spectra recorded for the twill differ from that of the single yarns, with an $f_{c} \approx 1 \mathrm{~Hz}$ (Fig. S9, Suppl. Information). This makes the textile well suited for converting the mechanical energy from human motion, e.g., walking, into electrical energy. Again, an equivalent electrical circuit can be used to predict the generated output. We measured the resistance and capacitance of the textile bands with the LCR meter connected to the textile electrodes (Table S1, Suppl. Information). For simulations, the intrinsic $V_{i}$ was selected so that each circuit model produced the same voltage amplitude $V_{\text {out }}$ (Fig. S10, Suppl. Information) with a load impedance $Z_{\text {load }}$ equivalent to the internal impedance of the measurement device (cf. equation 1). Next, we could predict the generated active power $P$ :

$P=\frac{V_{\mathrm{p}} I_{\mathrm{p}}}{2} \cos \theta$

where $V_{\mathrm{p}}$ is the peak voltage, $I_{\mathrm{p}}$ is the peak current and $\theta$ is the phase angle, for several different values of $R_{\text {load }}$ in the circuit 


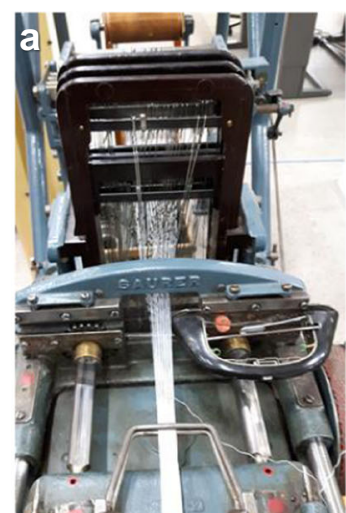

d plain weave

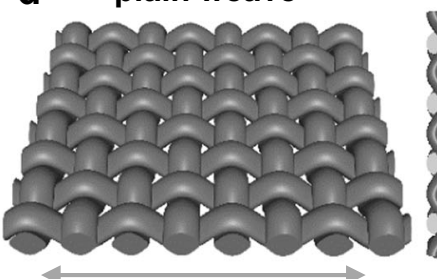

conducting weft

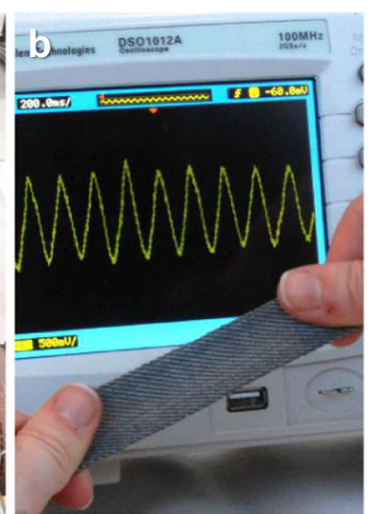

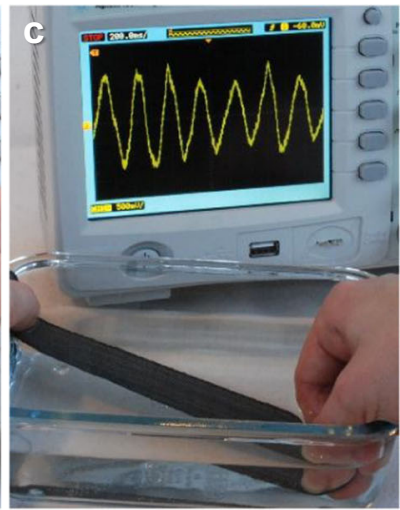

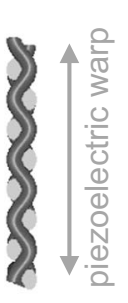

e twill

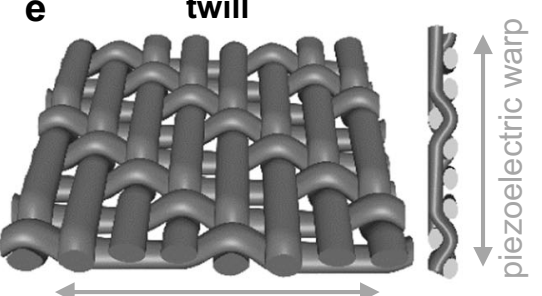

conducting weft
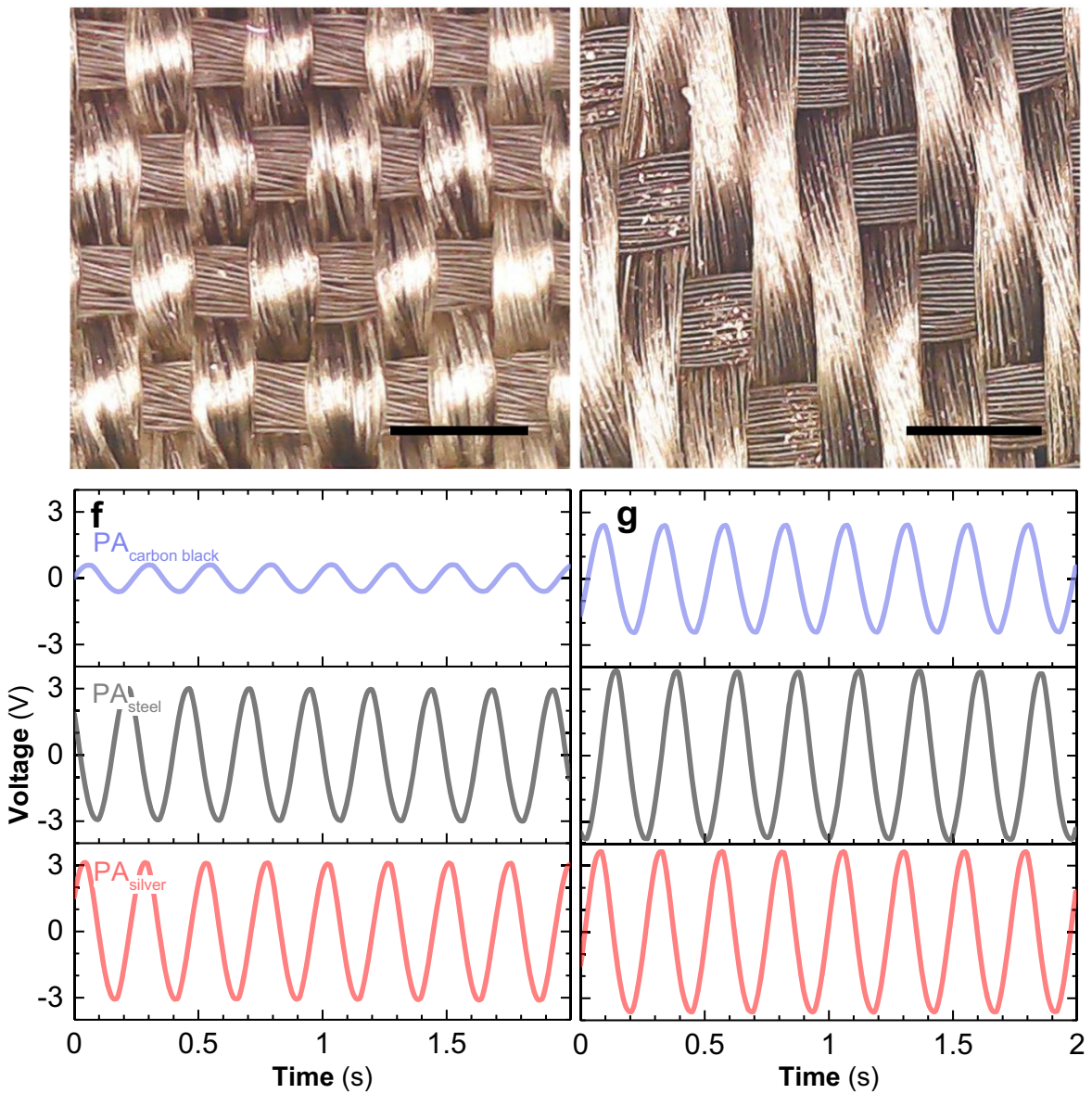

Fig. 3 Manufacture and piezoelectric behaviour of woven bands. a Photograph of the band weaving machine used for production of the piezoelectric textiles. Photographs of the textile bands (b: dry; c: submerged in water) when stretched by hand: the output voltage is shown on the oscilloscope screen. Schematics (top) and photographs (bottom, scale bars are $1 \mathrm{~mm}$ ) of produced (d) plain weave and (e) twill textile

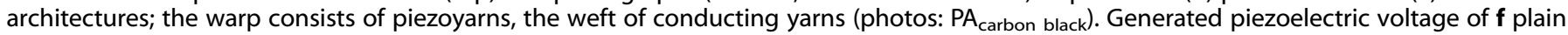
weave and $\mathbf{g}$ twill textiles at $f=4 \mathrm{~Hz}$ and $\varepsilon=0.25 \%$ : outer electrodes are $\mathrm{PA}_{\text {carbon black }}$ (blue), $\mathrm{PA}_{\text {steel }}\left(\right.$ grey), and $\mathrm{PA}_{\text {silver }}($ red) 


\section{6}

a

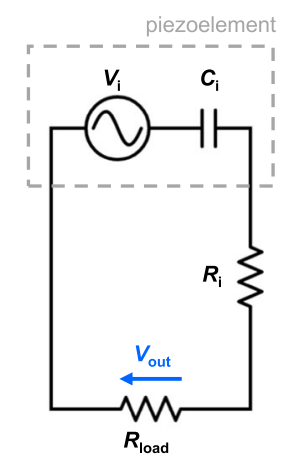

c

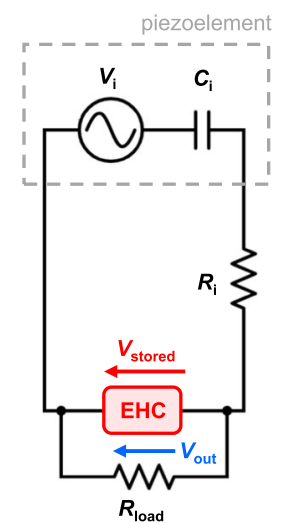

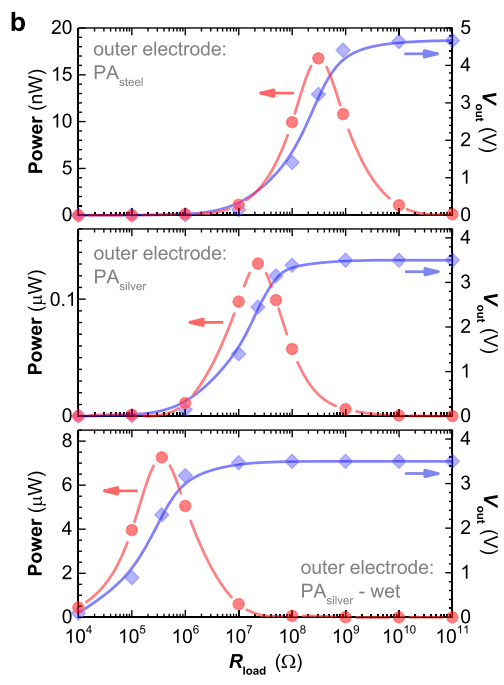

d

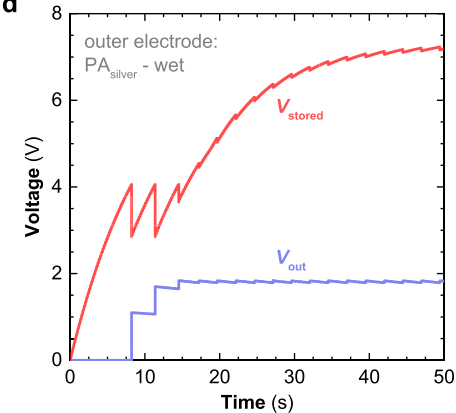

Fig. 4 Equivalent circuits of piezoelectric textiles, and predicted output voltage and power. a Equivalent electric circuit of the woven piezoelectric textiles with conducting yarns as outer electrodes. b Predicted output peak voltage $V_{\text {out }}$ (blue) and power (red) of textiles produced with $\mathrm{PA}_{\text {steel }}$ (top: $V_{\mathrm{i}}=4.7 \mathrm{~V} ; C_{i}=0.26 \mathrm{nF} ; R_{\mathrm{i}}=4935 \mathrm{k} \Omega$ ); $\mathrm{PA}_{\text {silver }}$ (centre: $V_{\mathrm{i}}=3.5 \mathrm{~V} ; C_{i}=3.5 \mathrm{nF} ; R_{\mathrm{i}}=100 \mathrm{k} \Omega$ ); and $\mathrm{PA}_{\text {silver }}$-wet (bottom: $V_{\mathrm{i}}=3.5 \mathrm{~V} ; C_{\mathrm{i}}=220 \mathrm{nF} ; R_{\mathrm{i}}=41 \mathrm{k} \Omega$ ); $C_{\mathrm{i}}$ and $R_{\mathrm{i}}$ were measured, $V_{\text {out }}$ and the power were modelled with SPICE software LTspice. c Equivalent electric circuit for a piezoelectric textile connected to an energy harvesting circuit (EHC). d Predicted charging voltage stored in the EHC, $V_{\text {stored }}$ (red), and output voltage $V_{\text {out }}$ (blue); outer electrodes $\mathrm{PA}_{\text {silver }}$-wet $\left(V_{\mathrm{i}}=3.5 \mathrm{~V} ; C_{\mathrm{i}}=220 \mathrm{nF} ; R_{\mathrm{i}}=41 \mathrm{k} \Omega ; R_{\text {load }}=1\right.$ $\mathrm{M} \Omega$ )

model (Fig. 4a). The maxima occur where $R_{\text {load }}=Z_{\mathrm{i}}$, here calculated for $f=2 \mathrm{~Hz}$. Increasing the values for $R_{\text {load }}$ results in a higher output voltage (approaching $V_{\mathrm{i}}$ ) but lower generated power (Fig. 4b). We find that the output power of twill textiles varies by nearly one order of magnitude when changing the material of the outer electrode yarns, because of differences in $Z_{i}$. The calculated maximum power for the $\mathrm{PA}_{\text {steel }}$ electrode is $16 \mathrm{nW}$ whereas the fabric with a $\mathrm{PA}_{\text {silver }}$ electrode yarn is predicted to generate a power of up to $130 \mathrm{nW}$.

Gratifyingly, adding water to the outer surface of the band with $\mathrm{PA}_{\text {silver }}$ weft both reduces its resistance and significantly increases its inherent capacitance (Table S1, Suppl. Info), thus reducing $Z_{\mathrm{i}}$ from $23 \mathrm{M} \Omega$ to $360 \mathrm{k} \Omega$. The increase in capacitance is due to geometrical factors. Each piezoelectric fibre constitutes a cylindrical capacitor with a capacitance $C$ :

$C=\frac{\varepsilon_{r} \varepsilon_{0} A}{r_{2} \ln \frac{r_{2}}{r_{1}}}$

where $\varepsilon_{\mathrm{r}}$ is the dielectric constant of the insulator (PVDF), $\varepsilon_{0}$ is the permittivity of vacuum, $A$ is the area of the outer electrode and $r_{1}$ and $r_{2}$ are the radius of the conducting core and the entire bicomponent fibre, respectively. The addition of a conducting liquid to the surface of the textile results in a substantial increase in contact area between the piezoyarn (consisting of 24 fibres) and the conducting yarns (cf. Fig. S11, Suppl. Information). According to equation 3 the substantial increase in outer electrode area leads to a higher capacitance (cf. characterisation of yarns with silver paste as the outer electrode). Due to the decrease in impedance, according to our model a textile band with $\mathrm{PA}_{\text {silver }}$ yarns + water as the outer electrode can produce an output power exceeding $7 \mu \mathrm{W}$ (Fig. 4b).

Energy harvesting with piezoelectric textiles

In a final set of experiments we explored the use of our piezoelectric textiles for energy harvesting. We connected an energy harvesting circuit (EHC) including a rectifier and an energy storing capacitor of $22 \mu \mathrm{F}$ to the textile. The EHC represents a high impedance interface and provides an output voltage of $1.6-2 \mathrm{~V}$ to the load (we used a $1 \mathrm{M} \Omega$-resistor) after a certain charging time of the built-in capacitor. The EHC provides a DC voltage, thus the supplied power is calculated as:

$P_{\text {EHC }}=\frac{V_{\text {out }}^{2}}{R_{\text {load }}}$

Modelling of the combined piezoelectric plus energy harvesting textile indicates that a continuous output power $P_{\mathrm{EHC}}$ of $4 \mu \mathrm{W}$ can be maintained after an initial charging time of less than $15 \mathrm{~s}$ (Fig. 4c, d and Fig. S12, Suppl. Information). To explore this design in practice, we modified a commercial laptop case by replacing a 20 $\mathrm{cm}$ length of its shoulder strap by the piezoelectric twill/PA $\mathrm{A}_{\text {silver }}$ band (Fig. 5a). Weights (a Macmillan English dictionary and several polymer textbooks) were placed in the bag to ensure a pre-load on the strap corresponding to $30 \mathrm{~N}$. After wetting the strap, stairwalking with the bag over the shoulder produced a peak output of up to $8 \mathrm{~V}$ (Fig. 5b, c). After an initial charging period of approximately $1 \mathrm{~min}$ we were able to produce an average output voltage of $1 \mathrm{~V}$, corresponding to an average output power $P_{\mathrm{EHC}}=$ $1 \mu \mathrm{W}$ for as long as walking continued. A 2-min staircase climb generated an electrical energy $W \approx 0.4 \mathrm{~mJ}$ (Fig. $5 \mathrm{~d}$ ), calculated as:

$W=W_{\text {stored }}+W_{\text {out }}=\frac{C V_{\text {stored }}^{2}}{2}+t \cdot \frac{V_{\text {out }}^{2}}{R_{\text {load }}}$

where $W_{\text {stored }}$ is the energy stored in the capacitor, $W_{\text {out }}$ is the energy drawn from the $\mathrm{EHC}, C$ is the capacitance of the storage capacitor, $V_{\text {stored }}$ is the voltage across this capacitor, $V_{\text {out }}$ is the voltage over the load of resistance $R_{\text {load }}$ and $t$ is time. Assuming the walking pace to be two steps per second, the average energy generated is about $2 \mu \mathrm{J}$ per step.

As the type of motion that the wearer exerts on the piezoelectric textile affects its output, we monitored the generated voltage from a stair-walking motion while carrying the laptop case either by hand or across the shoulder. The piezoyarns' high sensitivity to tensile deformation is apparent from the generated output voltage pattern. When carrying the bag on the left shoulder, footsteps with the left foot produce voltage peaks twice as high as steps with the right foot (Fig. S13, Suppl. Information). Carrying the bag by hand (as in Fig. 5a) instead produces the same peak voltage at a steadier pace (Fig. S12c, Suppl. Information), significantly increasing the energy produced to more than $1 \mathrm{~mJ}$ after $50 \mathrm{~s}$ (Fig. 5e). This provides a continuous power of $4 \mu \mathrm{W}$, corresponding to $1.9 \mu \mathrm{W} / \mathrm{cm}^{3}$ or 3.3 $\mu \mathrm{W}$ per gram piezoyarn, after an initial charging time of less than $15 \mathrm{~s}$. The output voltage is in good agreement with the value that we predict based on the equivalent electric circuit model (in Fig. 4d). To demonstrate use of the harvested energy, we replaced the resistive load with a light emitting diode (LED), which draws $1 \mathrm{~mA}$ at $1.6 \mathrm{~V}$. The generated power from the shoulder strap is sufficient 
a

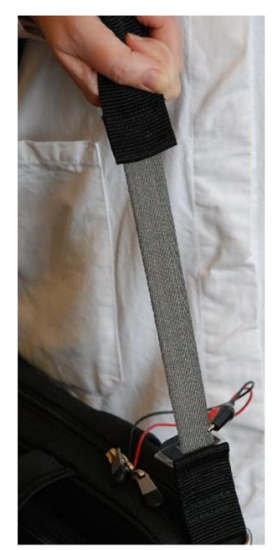

b
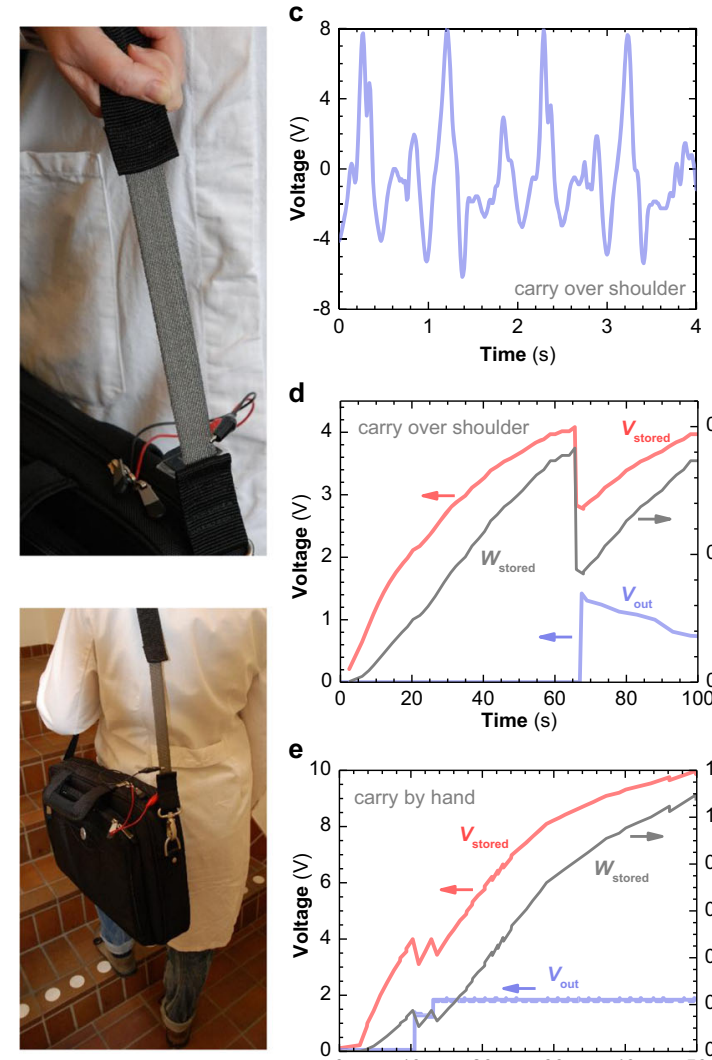

d
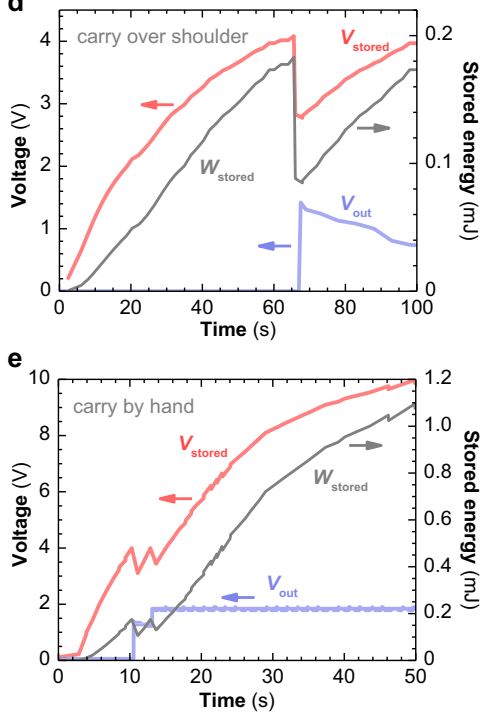

f

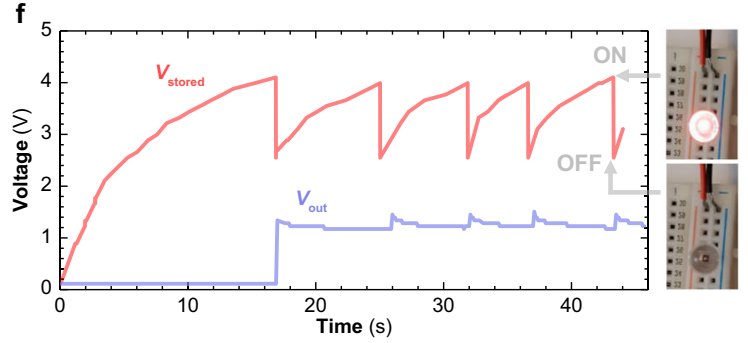

Fig. 5 Energy harvesting with a piezoelectric shoulder strap. a A twill-woven textile band with $\mathrm{PA}_{\text {silver }}$ conducting yarn as the outer electrode was sewn into the shoulder strap of a laptop case. b Walking up and down stairs with the laptop bag over the shoulder generates $\mathrm{c}$ a piezoelectric voltage where the peaks correspond to footfall. $\mathbf{d}$ The voltage $V_{\text {stored }}(\mathrm{red})$ and energy $W_{\text {stored }}$ (grey) stored in an EHC, and the output voltage $V_{\text {out }}$ (blue) when carrying the laptop case over the shoulder. An average output power of $1 \mu \mathrm{W}$ is obtained after an initial charging time of about $1 \min \left(R_{\text {load }}=1 \mathrm{M} \Omega\right)$. e $V_{\text {stored }}\left(\right.$ red), $W_{\text {stored }}$ (grey) and $V_{\text {out }}$ (blue) when holding the laptop case by hand. The piezoelectric textile continuously generates $4 \mu \mathrm{W}$ after an initial charging time of $15 \mathrm{~s}$. f The load resistor (as in e) can be replaced with an LED, which then blinks continuously after an initial charging time of about $15 \mathrm{~s}$. The results presented in c-f were produced under wet conditions

to periodically drive the LED, resulting in continuous blinking at a frequency of on average $0.13 \mathrm{~Hz}$ (Fig. 5f).

\section{DISCUSSION}

We have presented a fully textile piezoelectric generator and demonstrated its practical use for everyday unobtrusive energy harvesting. The piezoelectric textile was produced using low cost materials and manufactured with readily scalable textile manufacturing methods. In a practical example a $2.5 \mathrm{~cm} \times 20 \mathrm{~cm}$ piece of the textile, used as the shoulder strap of a laptop case, produced a continuous output of $4 \mu \mathrm{W}$. Assuming that the generated power scales linearly with area, fabricating the entire strap and the load carrying part of the laptop case from the piezoelectric textile $\left(\approx 1000 \mathrm{~cm}^{2}\right)$ would result in a generated power approaching $0.1 \mathrm{~mW}$, sufficient to power several RFID tags or wireless sensor nodes. ${ }^{54}$ In further developments of the piezoyarns, an increase in conductivity of the core electrode to $\sim 20 \mathrm{~S} / \mathrm{cm}$, e.g., by replacing carbon black with carbon nanotubes or graphene, is anticipated to provide a further 100 -fold increase in generated power to $10 \mathrm{~mW}$, which is needed for e.g., a Bluetooth transceiver. Likewise, decreasing the resistance of the outer electrode by increasing the yarn conductivity or simply adding more yarn, would further enhance the power output. The use of a conducting coating as the outer electrode would have the advantage of reducing the contact resistance towards the piezoyarns. The development of such a coating presents a considerable challenge, as its practical use would require a high abrasion resistance and thus an affinity to the chemically inert PVDF.

The here presented manufacturing route allows electrical functionality to be added while maintaining desirable textile qualities such as flexibility, drape, tensile strength and ease of processing (e.g., sewing). In future textile applications, large area woven piezoelectric fabrics may be used in e.g., hammocks, shoe soles, in the upholstery of passenger vehicles or as filler in structural composites, to convert ambient vibrations in combination with biomechanical movements from the user to electrical energy. Such e-textile formats can be anticipated to produce relevant levels of electrical power, without loss of structural functionality. Overall, our results point to textile materials and processes with high potential as a platform for electronic materials and macroelectronic structures with a broad range of functionalities.

\section{METHODS}

Fibre spinning

The materials used are PVDF: Solef 1006 (Solvay) with melt flow index MFI $\left(2.16 \mathrm{~kg}, 230^{\circ} \mathrm{C}\right)=40 \mathrm{~g} / 10 \mathrm{~min}$, LLDPE: ASPUN $6835 \mathrm{~A}$ (Dow) with MFI (2.16 $\mathrm{kg}, 190^{\circ} \mathrm{C}$ ) $=17 \mathrm{~g} / 10 \mathrm{~min}$, carbon black: Ketjenblack EC-600 JD (Akzo Nobel). The conducting core consists of LLDPE with $10 \mathrm{wt} \%$ carbon black, mixed/compounded in a twin screw extruder (Coperion ZSK $26 \mathrm{~K} \mathrm{10.6).}$ Fibre spinning was carried out in a pilot spinning line (Extrusion systems Ltd.) through a spinneret with 24 holes, each with a diameter of $0.6 \mathrm{~mm}$ and a land length of $1.2 \mathrm{~mm}$. For further details we refer to Refs. ${ }^{50,55}$

\section{Weaving}

Weaving was carried out on a band weaving loom, Saurer 60B 1-2, with a cam shedding system. The warp was 60 piezoyarns thread with one yarn per heddle eye, and 2 yarns per dent in a $10 \mathrm{dent} / \mathrm{cm}$ reed. The average speed was $180 \mathrm{picks} / \mathrm{min}$, corresponding to $\sim 16 \mathrm{~cm}$ woven $\mathrm{band} / \mathrm{min}$. The bands were $2.5 \mathrm{~cm}$ wide and each approximately $1 \mathrm{~m}$ long, with an average thickness of $0.43 \mathrm{~mm}$. The weft yarns were: a polyamide yarn commingled with $20 \%$ thin stainless-steel staple fibres (Bekintex BK 50/2 from Bekaert), a silver plated polyamide yarn (Shieldex from Statex) and a polyamide yarn coated with carbon black-particles (Shakespeare F9416 from Resistat).

Poling

Poling was carried out in an oven at $70^{\circ} \mathrm{C}$. The core electrodes were connected by (for single yarns) cutting the fibre ends with a razor blade and covering them with silver paint (Agar Scientific) or by (for warp yarns in woven bands) melt pressing the fibre ends sandwiched between two films of a $10 \mathrm{wt} \%$ carbon black/polyethylene composite at $135^{\circ} \mathrm{C}$. In contact poling the yarn was connected to a high voltage supply (Gamma High Voltage) set to $1.6 \mathrm{kV}$ for $10 \mathrm{~s}$. In corona poling the core electrodes were grounded, and the second poling electrode was constituted by the 
needles in a custom-made corona poling device, connected to a high voltage supply set to $7 \mathrm{kV}$. Corona poling was carried out for $3 \mathrm{~min}$. All samples were short-circuited between aluminium sheets after poling to remove excess surface charge.

\section{Wide-angle X-ray scattering (WAXS)}

WAXS measurements were performed at the D-line, Cornell High Energy Synchrotron Source (CHESS) at Cornell University. The X-ray beam with a wavelength of $1.162 \AA$ and size of about $0.5 \mathrm{~mm}$ was directed to the sample. A Pilatus $200 \mathrm{k}$ detector located $169.5 \mathrm{~mm}$ from the sample was used to capture the diffractogram with an exposure time of $1 \mathrm{~s}$.

\section{Mechanical characterisation}

Tensile tests and dynamic electromechanical characterisation of the piezoyarns was carried out in a TA Instruments DMA Q800 (Waters) using a pre-load of $0.5 \mathrm{~N}$. For dynamic electromechanical characterisation of the bands a servo-hydraulic tensile tester MTS 66-21B-01(MTS Systems) was used with a pre-load of $30 \mathrm{~N}$ and a gauge length of $100 \mathrm{~mm}$. A sinusoidal strain was applied with a (1) strain amplitude of $0.1 \%$ while ramping the frequency from 0.1 to $10 \mathrm{~Hz}$, or (2) strain amplitude of $0.25 \%$ at $4 \mathrm{~Hz}$.

\section{Electrical characterisation and modelling}

Impedance was measured using a Keysight U1731C LCR meter set to series mode and $f=100 \mathrm{~Hz}$. The output voltage of the piezoyarns and the laptop case strap was measured with a digital oscilloscope Picoscope 5444 (Pico Technology) with an input resistance $10 \mathrm{M} \Omega$ and input capacitance $13 \mathrm{pF}$. The output voltage of the woven bands was recorded using a NI DAQPad6016 (National Instruments) with an input resistance $100 \mathrm{G} \Omega$ and input capacitance $100 \mathrm{pF}$. The electrical circuit calculations were carried out using the free simulation software LTspice XVII(x64) (Linear Technology).

\section{Energy harvesting}

The piezoelectric energy harvesting power supply circuit LTC3588EMSE-1/2 (Linear Technology) was used as mounted on a demo board with storage capacitor included. The stair-walking motions were carried out by (1) carrying the laptop case over the shoulder and stepping up and down an aerobic exercise stepper platform of $20 \mathrm{~cm}$ height, and (2) by holding the laptop case by the hand and gently bouncing it up and down. For characterisation in the wet state, tap water was applied to the textile surface until it was moist but not dripping. The tap water was drawn at Chalmers, Göteborg, during spring 2017 and is reported to have a median conductivity of $0.20 \mathrm{mS} / \mathrm{cm}^{56}$

Data availability statement

All relevant data are available from the authors upon request.

\section{ACKNOWLEDGEMENTS}

The authors thank senior research engineer Roger Sagdahl, Chalmers University of Technology, for valuable discussions on energy harvesting. CHESS (supported by the NSF \& NIH/NIGMS via NSF award DMR-1332208) is gratefully acknowledged for providing experimental time for WAXS measurements. Financial support from the Swedish Foundation for Strategic Research (SSF) through grant no. SBE13-0086, the Knut and Alice Wallenberg Foundation through a Wallenberg Academy Fellowship, and the European Research Council (ERC) under grant agreement no. 637624 is gratefully acknowledged.

\section{AUTHOR CONTRIBUTIONS}

A. L., E. N. and B. H. developed the piezoelectric yarns. K. R. planned and performed the weaving. K. R. and E. N. characterised the woven textiles. L. Y. carried out the WAXS-characterisation and analysis. A. L. planned and performed the characterisation of piezoyarns, modelling, fabrication and evaluation of the textile energy harvesting device. A. L. and C. M. analysed the results and prepared the manuscript.

\section{ADDITIONAL INFORMATION}

Supplementary information accompanies the paper on the npj Flexible Electronics website (https://doi.org/10.1038/s41528-018-0022-4).
Competing interests: The authors declare no competing financial interests.

Publisher's note: Springer Nature remains neutral with regard to jurisdictional claims in published maps and institutional affiliations.

\section{REFERENCES}

1. Zeng, W. et al. Fiber-based wearable electronics: a review of materials, fabrication, devices, and applications. Adv. Mater. 26, 5310-5336 (2014).

2. Hu, L. \& Cui, Y. Energy and environmental nanotechnology in conductive paper and textiles. Energy Environ. Sci. 5, 6423 (2012).

3. Post, E. R., Orth, M., Russo, P. R. \& Gershenfeld, N. E-broidery design and fabrication of textile-based computing. IBM Syst. J. 39, 840-860 (2000).

4. Takamatsu, S. et al. Wearable keyboard using conducting polymer electrodes on textiles. Adv. Mater. 28, 4485-4488 (2016).

5. Zirkl, M. et al. An all-printed ferroelectric active matrix sensor network based on only five functional materials forming a touchless control interface. Adv. Mater. 23, 2069-2074 (2011).

6. Seyedin, S. et al. Knitted strain sensor textiles of highly conductive all-polymeric fibers. ACS Appl. Mater. Interfaces 7, 21150-21158 (2015).

7. Cherenack, K. \& van Pieterson, L. Smart textiles: challenges and opportunities. J. Appl. Phys. 112, 091301 (2012).

8. Zhao, Z. et al. Machine-washable textile triboelectric nanogenerators for effective human respiratory monitoring through loom weaving of metallic yarns. Adv. Mater. 28, 10267-10274 (2016).

9. Ashok Kumar, L. \& Vigneswaran, C. Electronics in Textiles and Clothing-Design, Products and Applications. (Taylor \& Francis Group, LLC, Boca Raton, FL, US, 2016).

10. Hamedi, M., Forchheimer, R. \& Inganäs, O. Towards woven logic from organic electronic fibres. Nat. Mater. 6, 357-362 (2007).

11. Jo, A. et al. Textile resistance switching memory for fabric electronics. Adv. Funct. Mater. 27, 1605593 (2017).

12. Weng, W., Chen, P., He, S., Sun, X. \& Peng, H. Smart electronic textiles. Angew. Chem. Int. Ed. 55, 6140-6169 (2016).

13. Bauer, S. et al. 25th anniversary article: a soft future: from robots and sensor skin to energy harvesters. Adv. Mater. 26, 149-161 (2014).

14. Haines, C. S. et al. Artificial muscles from fishing line and sewing thread. Science 343, 868-872 (2014).

15. Maziz, A. et al. Knitting and weaving artificial muscles. Sci. Adv. 3, e1600327 (2017).

16. Peng, H. Fiber-Shaped Energy Harvesting and Storage Devices. (Springer, Berlin, Heidelberg, 2015)

17. Wen, Z. et al. Self-powered textile for wearable electronics by hybridizing fibershaped nanogenerators, solar cells, and supercapacitors. Sci. Adv. 2, e1600097 (2016).

18. Ryan, J. D., Mengistie, D. A., Gabrielsson, R., Lund, A. \& Müller, C. Machinewashable PEDOT:PSS dyed silk yarns for electronic textiles. ACS Appl. Mater. Interfaces 9, 9045-9050 (2017).

19. Du, Y. et al. Thermoelectric fabrics: toward power generating clothing. Sci. Rep. 5, 6411 (2015).

20. Lee, J. A. et al. Woven-yarn thermoelectric textiles. Adv. Mater. 28, 5038-5044 (2016).

21. Kim, S. J., We, J. H. \& Cho, B. J. A wearable thermoelectric generator fabricated on a glass fabric. Energy Environ. Sci. 7, 1959 (2014).

22. Chen, J. et al. Micro-cable structured textile for simultaneously harvesting solar and mechanical energy. Nat. Energy 1, 16138 (2016).

23. Kim, K. N. et al. Highly stretchable 2D fabrics for wearable triboelectric nanogenerator under harsh environments. ACS Nano 9, 6394-6400 (2015).

24. Fan, F. R., Tang, W. \& Wang, Z. L. Flexible Nanogenerators for Energy Harvesting and Self-Powered Electronics. Adv. Mater. 28, 4283-4305 (2016).

25. Hu, F., Cai, Q., Liao, F., Shao, M. \& Lee, S. T. Recent advancements in nanogenerators for energy harvesting. Small 11, 5611-5628 (2015).

26. Egusa, S. et al. Multimaterial piezoelectric fibres. Nat. Mater. 9, 643-648 (2010).

27. Lu, X., Qu, H. \& Skorobogatiy, M. Piezoelectric microstructured fibers via drawing of multimaterial preforms. Sci. Rep. 7, 2907 (2017).

28. $\mathrm{Pu}, \mathrm{X}$. et al. Wearable textile-based in-plane microsupercapacitors. Adv. Energy Mater. 6, 1601254 (2016).

29. Zhu, J. et al Wearable high-performance supercapacitors based on silversputtered textiles with $\mathrm{FeCO}_{2} \mathrm{~S}_{4}-\mathrm{NiCO}_{2} \mathrm{~S}_{4}$ composite nanotube-built multitripod architectures as advanced flexible electrodes. Adv. Energy Mater. 7, 1601234 (2016).

30. Jost, K., Dion, G. \& Gogotsi, Y. Textile energy storage in perspective. J. Mater. Chem. A 2, 10776 (2014) 
31. Fu, Y. et al. Integrated power fiber for energy conversion and storage. Energy Environ. Sci. 6, 805 (2013).

32. Katsouras, I. et al. The negative piezoelectric effect of the ferroelectric polyme poly(vinylidene fluoride). Nat. Mater. 15, 78-84 (2016).

33. Lovinger, A. J. Ferroelectric polymers. Science 220, 1115-1121 (1983).

34. Martín, J. et al. Solid-state-processing of $\delta$-PVDF. Mater. Horiz. 4, 408-414 (2017).

35. Kymissis, J., Kendall, C., Paradiso, J. \& Gershenfeld, N. Parasitic power harvesting in shoes. IEEE 2nd Int. Symp. Wearable Comp. 2-9 (1998).

36. Granstrom, J., Feenstra, J., Sodano, H. A. \& Farinholt, K. Energy harvesting from a backpack instrumented with piezoelectric shoulder straps. Smart Mater. Struct. 16, 1810-1820 (2007).

37. Baji, A., Mai, Y. W., Li, Q. \& Liu, Y. Electrospinning induced ferroelectricity in poly (vinylidene fluoride) fibers. Nanoscale 3, 3068-3071 (2011).

38. Persano, L. et al. High performance piezoelectric devices based on aligned arrays of nanofibers of poly(vinylidenefluoride-co-trifluoroethylene). Nat. Commun. 4 , 1633 (2013).

39. Chang, J., Dommer, M., Chang, C. \& Lin, L. Piezoelectric nanofibers for energy scavenging applications. Nano Energy 1, 356-371 (2012).

40. Fang, J., Wang, X. \& Lin, T. Electrical power generator from randomly oriented electrospun poly(vinylidene fluoride) nanofibre membranes. J. Mater. Chem. 21, 11088 (2011).

41. Soin, N. et al. Novel "3-D spacer" all fibre piezoelectric textiles for energy harvesting applications. Energy Environ. Sci. 7, 1670 (2014).

42. Ziabicki, A. Fundamentals of Fibre Formation. (Wiley, Bath, UK, 1976)

43. Lund, A. \& Hagström, B. Melt spinning of poly(vinylidene fluoride) fibers and the influence of spinning parameters on $\beta$-phase crystallinity. J. Appl. Polym. Sci. 116 2685-2693 (2010).

44. Lund, A. \& Hagström, B. Melt spinning of $\beta$-phase poly(vinylidene fluoride) yarns with and without a conductive core. J. Appl. Polym. Sci. 120, 1080-1089 (2011).

45. Steinmann, W. et al. Structure, properties, and phase transitions of melt-spun poly(vinylidene fluoride) fibers. J. Appl. Polym. Sci. 120, 21-35 (2011).

46. Wan, C. \& Bowen, C. R. Multiscale-structuring of polyvinylidene fluoride for energy harvesting: the impact of molecular-, micro- and macro-structure. J. Mater. Chem. A 5, 3091-3128 (2017)

47. Strååt, M., Toll, S., Boldizar, A., Rigdahl, M. \& Hagström, B. Melt spinning of conducting polymeric composites containing carbonaceous fillers. J. Appl. Polym. Sci. 119, 3264-3272 (2011)
48. White, J. L. \& Tanaka, H. Elongational Flow and Melt-Spinning Instability of Concentrated Suspensions of Small Particles in Polymer Melts. J. Appl. Polym. Sci. 26, 579-589 (1981).

49. Zoller, P. \& Walsh, D. J. Standard Pressure-volume-temperature Data For Polymers. (Technomic Publishing Inc., Lancaster, PA, US, 1995).

50. Nilsson, E., Lund, A., Jonasson, C., Johansson, C. \& Hagström, B. Poling and characterization of piezoelectric polymer fibers for use in textile sensors. Sens. Act. A Phys. 201, 477-486 (2013).

51. Åkerfeldt, M., Lund, A. \& Walkenström, P. Textile sensing glove with piezoelectric PVDF fibers and printed electrodes of PEDOT:PSS. Text. Res. J. 85, 1789-1799 (2015).

52. Starner, T. Human-powered wearable computing. IBM Syst. J. 35, 618-629 (1996)

53. Hagström, B., Lund, A. \& Nilsson, E. Method of producing a piezoelectric and pyroelectric fiber. WO 2014/161920 A1 (2014).

54. Vullers, R. J. M., van Schaijk, R., Doms, I., Van Hoof, C. \& Mertens, R. Micropower energy harvesting. Solid State Electron. 53, 684-693 (2009).

55. Lund, A., Jonasson, C., Johansson, C., Haagensen, D. \& Hagström, B. Piezoelectric polymeric bicomponent fibers produced by melt spinning. J. Appl. Polym. Sci. 126, 490-500 (2012)

56. Information about water quality. http://goteborg.se/wps/portal/start/vatten-ochavlopp/dricksvatten/dricksvattnets-kvalitet. (City of Göteborg, Sweden, 2017).

Open Access This article is licensed under a Creative Commons C. Attribution 4.0 International License, which permits use, sharing, adaptation, distribution and reproduction in any medium or format, as long as you give appropriate credit to the original author(s) and the source, provide a link to the Creative Commons license, and indicate if changes were made. The images or other third party material in this article are included in the article's Creative Commons license, unless indicated otherwise in a credit line to the material. If material is not included in the article's Creative Commons license and your intended use is not permitted by statutory regulation or exceeds the permitted use, you will need to obtain permission directly from the copyright holder. To view a copy of this license, visit http://creativecommons. org/licenses/by/4.0/.

(c) The Author(s) 2018 